\title{
Effectiveness of intra-articular ozone injections on outcomes of post-arthroscopic surgery for knee osteoarthritis
}

\author{
XIANGJIANG WANG ${ }^{1,2}$, GUIQING WANG $^{2}$, CHUNLEI LIU $^{2}$ and DAOZHANG CAI ${ }^{1}$ \\ ${ }^{1}$ Department of Orthopedics, The Third Affiliated Hospital of Southern Medical University, Guangzhou, \\ Guangdong 510630; ${ }^{2}$ Department of Orthopedics, The Sixth Affiliated Hospital of Guangzhou Medical University, \\ Qingyuan, Guangdong 511508, P.R. China
}

Received December 15, 2017; Accepted March 9, 2018

DOI: $10.3892 /$ etm.2018.6101

\begin{abstract}
The purpose of the present study was to evaluate intra-articular ozone injection following arthroscopic surgery for knee osteoarthritis (OA) with regard to its efficacy in pain reduction, joint function and quality of life improvement. The present study retrospectively evaluated 80 patients with symptomatic knee OA (Kellgren-Lawrence grade II or III), who either did or did not receive $20 \mathrm{ml}$ of $20 \mu \mathrm{g} / \mathrm{ml}$ ozone as an intra-articular injection after arthroscopic surgery. The minimum follow-up period was 12 months. The outcomes evaluated for knee OA were pain on the Visual Analogue Scale (VAS), Lequesne Index, Western Ontario and McMaster Universities Osteoarthritis Index (WOMAC) and Clinical Global Impression (CGI). The VAS score in the ozone group was significantly better than that in the control group at all post-operative follow-up time-points $(\mathrm{P}<0.05)$. The ozone group also exhibited a significantly greater improvement in Lequesne Index scores $(\mathrm{P}<0.05)$. In the ozone group, the score on the WOMAC-pain, WOMAC-stiffness and WOMAC-function subscales, as well as the total WOMAC score decreased significantly $(\mathrm{P}<0.05)$. Furthermore, in the ozone group a significantly higher number of patients $(\mathrm{P}<0.05)$ with better CGI grades was encountered compared with that in the control group at the 12-month follow-up assessment, despite comparable baseline values in all aforementioned clinical measures between the two groups of patients. The present study suggests that intra-articular ozone injections after arthroscopic surgery may effectively improve the outcomes of arthroscopic surgery in terms of pain relief, functional improvement and quality of life in patients with knee OA of Kellgren-Lawrence grade II or III.
\end{abstract}

Correspondence to: Dr Daozhang Cai, Department of Orthopedics, The Third Affiliated Hospital of Southern Medical University, 183 West Zhongshan Road, Tianhe, Guangzhou, Guangdong 510630, P.R. China

E-mail: daozhang@medmail.com

Key words: intra-articular, ozone, arthroscopy, knee osteoarthritis

\section{Introduction}

Knee osteoarthritis (OA) is a common disease associated with tissue inflammation, physical disability and imbalanced homeostasis in the cartilage. Approximately $25 \%$ of people aged $>50$ years experience knee pain, articular stiffness and decreased function due to knee OA (1). At present, various types of surgery are available for the treatment of knee OA. Arthroscopic surgery is a minimally invasive surgical procedure and is advocated as one treatment option for symptomatic relief. The procedure includes lavage, partial meniscectomy, limited synovectomy, excision of osteophytes, removal of loose bodies and adhesiolysis $(2,3)$. The curative effect is satisfactory; however, the long-term efficacy of arthroscopic surgery in the treatment of knee OA has remained a topic of controversy amongst clinicians $(4,5)$, since the surgery may remove cartilage fragments, mechanical irritations, inflammatory cells and other factors from the joint, but cannot reverse or repair the knee OA. Therefore, numerous investigators have sought solutions combining arthroscopic surgery and viscosupplementation, including steroids and hyaluronan (HA), to improve outcomes of knee OA (6-10). However, studies have suggested that the use of viscosupplementation to repair damaged articular cartilage has limited efficacy after arthroscopic surgery.

In the past decade, there has been an increasing interest in the use of ozone injection for the treatment of knee OA. Ozone is a soluble gas with high oxidative activity, and exerts an anti-nociceptive effect through several mechanisms (11). Several studies and clinical experiments have demonstrated that ozone therapy has positive effects in terms of reducing pain and inflammation, improving function and benefiting the trophism of bone and cartilage, increasing vascularization, and increasing repair of the articular and subchondral bone (12-14). Intra-articular ozone injections are an effective and low-cost procedure to control pain in knee OA. Based on this previous work, the aim of the present study was to investigate whether OA injections exert a beneficial effect on OA of the knee after arthroscopic surgery.

\section{Materials and methods}

Participants. This study was approved by the Ethics Committee of the Sixth Affiliated Hospital of Guangzhou 
Medical University and was performed in accordance with the provisions of the World Medical Association's Declaration of Helsinki 1995 (revised in Tokyo, 2004). Due to the retrospective nature of the study, the Ethics Committee of the Sixth Affiliated Hospital of Guangzhou Medical University waived the requirement for study participants to provide informed consent (15).

The inclusion criteria were as follows: i) Patients with knee OA (Kellgren-Lawrence Grade II or III) (16) with pain $\geq 4$ on the visual analogue scale (VAS) (17); ii) patients aged 60-80 years; iii) patients for whom any other conservative treatments had failed (non-anti-inflammatory steroidal drugs, rehabilitation or physical therapy); and iv) patients with stable knees without malalignment.

The exclusion criteria were as follows: i) Patients with severe OA or aged $<60$ or $>80$ years; ii) patients with any systemic, mental or neurological deficiency; iii) patients in whom any invasive procedure had been applied to the knee or any intra-articular injection had been administered prior to the treatment; and iv) patients with any formal contraindication to ozone therapy (thrombocytopenia, angiotensin-converting enzyme inhibitor treatment, favism, hyperthyroidism, serious cardiovascular instability or ozone) or a history of previous ozone therapy.

The participants of the present study were selected from a total of 110 patients who had undergone arthroscopic surgery at the Sixth Affiliated Hospital of Guangzhou Medical University (Qingyuan, China) between March 2011 and May 2014 and who were retrospectively evaluated. Among them, 90 patients had been diagnosed with mild-to-moderate or moderate knee OA (Kellgren-Lawrence Grade II or III) and met the enrolment criteria of the study. Following arthroscopic surgery, the patients were divided into two groups according to the type of treatment administered. In the ozone group, patients received $20 \mathrm{ml}$ of $20 \mu \mathrm{g} / \mathrm{ml}$ ozone as intra-articular injections after arthroscopic surgery, while another group who did not receive any intra-articular ozone injection served as a control group. The intra-articular ozone injections were performed once per week for 4 consecutive weeks, starting 2 weeks after surgery. The mean duration of follow-up was 13.7 months and the minimum follow-up duration was 12 months. In the ozone group, 6 patients were lost to follow-up and 4 cases were lost to follow-up in the control group. At the final follow-up, there were 42 patients in the ozone group and 38 in the control group. All arthroscopic surgeries and injections were performed by the same experienced physicians. Data on endpoints were collected prior to arthroscopic surgery and at 6 weeks and 3, 6 and 12 months after arthroscopic surgery. Demographic data and all post-operative outcome data were obtained from patient records (Fig. 1).

Arthroscopic surgical procedure. The standard arthroscopic debridement was performed under spinal or general anesthesia. The procedure in these patients included lavage of the joint with varying amounts of serum saline solution, and debridement of roughened surfaces with removal of loose debris. Meniscal tears were often resected and only loose unstable fragments were repaired, while carefully preserving as much meniscal tissue as possible. Loose bodies were removed as required. According to the protocol specifications, all patients received the same analgesic and exercise program after surgery. Celecoxib (200 mg) was administered orally as an analgesic supplement from postoperative $3 \mathrm{~h}$ and was repeated every $8 \mathrm{~h}$ until 2 days post-operation if the recorded VAS score was $\geq 4$. The total celecoxib consumption after the operation was also recorded.

Intra-articular injection procedure. The patient was placed in a supine position with the knee in an extended position. The knee to be punctured was subjected to an anti-sepsis procedure with iodine. After the patella had been located, its medial margin was pressed to subluxation in the lateral margin to increase the articular rim as much as possible. A 22-gauge needle was inserted along the superlateral margin of the patella into the suprapatellar pouch without ultrasound or other imaging guidance. Physicians ensured that the needle was not inside a blood vessel by aspirating the syringe. The ozone generator (Herrmann, Germany) was set to produce ozone $\left(\mathrm{O}_{3}\right)$ from $\mathrm{O}_{2}$ at a concentration of $20 \mu \mathrm{g} / \mathrm{ml}$. A $20-\mathrm{ml}$ syringe was connected to the exit of the generator and $20 \mathrm{ml}$ of the produced gas was drawn into the syringe. Before the injection of gas (18), $2 \mathrm{ml} \mathrm{1 \%} \mathrm{lidocaine} \mathrm{was} \mathrm{injected} \mathrm{into} \mathrm{the} \mathrm{knee.}$ Lidocaine was administered to reduce the burning pain felt in the knee during gas injection, which lasted several minutes.

Outcome measures. The efficacy of the treatment was evaluated using the VAS (17), Lequesne index (19), and Western Ontario and McMaster University Osteoarthritis Index (WOMAC) questionnaires (20). In the VAS, the proband selects a whole number on a scale of $0-10$, with 10 representing severe pain and 0 representing the absence of pain. The Lequesne index consists of 10 specific questions, including 5 items associated with pain or discomfort, 1 item associated with the maximum distance walked and 4 items associated with daily life activities. The score ranges from 0 to 24 points. The WOMAC scale consists of 24 questions that evaluate pain, stiffness and physical function during daily life activities, and each question has five response choices (none, mild, moderate, severe and extreme); the subtotal scores for pain (5 items), stiffness ( 2 items) and physical function (17 items) range from 0-20, 0-8 and 0-68, respectively, and the sum of all the points generates a value between 0 and 96 inclusive. Higher values indicate a worse patient condition. At the 12-month follow-up, the patient's overall impression of the treatment outcome was measured by a clinical global impression (CGI), including a 5-point ordinal scale (1, excellent; 2, good; 3, satisfactory; 4, unchanged; 5, worse).

Statistical analysis. Statistical analyses were performed using the SPSS version 19.0 software package (IBM Corp., Armonk, NY, USA). Values are expressed as the mean \pm standard deviation or sample proportions unless specified otherwise. To assess the normality of the data distributions, the data were compared using a Kolmogorov-Smirnov test. Quantitative data were compared between the two groups using an independent-samples t-test. The Chi-square test was used to assess the differences between the two groups in terms of qualitative data. To compare quantitative variables within each group prior to and after treatment, one-way analysis of variance with the LSD post hoc test was used. 


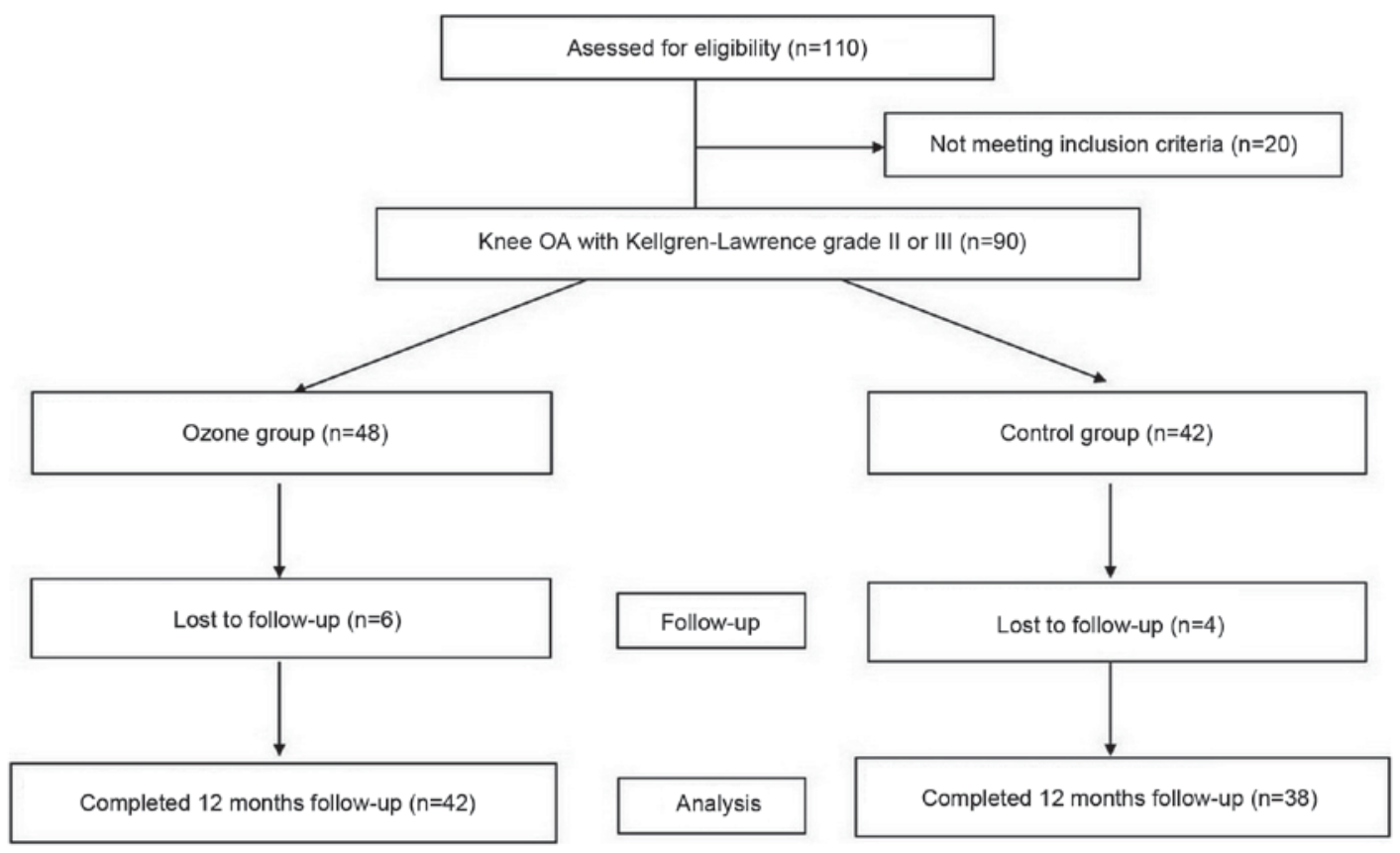

Figure 1. Flowchart of patient selection. OA, osteoarthritis.

$\mathrm{P}<0.05$ was considered to indicate a statistically significant difference.

\section{Results}

Demographic characteristics of the patients. A total of 80 patients (mean age, 71.5 years; age range, 60-80 years) with symptomatic knee OA (Kellgren-Lawrence grade II or III), comprising 42 patients in the ozone group and 38 in the control group, were enrolled in the present study. The two groups were comparable in terms of baseline demographics and disease profile, and there were no statistically significant differences between the groups (Table I). After ozone injection, 5 patients in the ozone group experienced a mild pain accompanied by erythema; the pain disappeared within a few minutes.

VAS, Lequesne index and WOMAC scores. Although the two groups had comparable baseline values, the VAS scores, Lequesne index scores, and the WOMAC-pain, WOMAC-stiffness, WOMAC-function and total WOMAC scores were significantly better in the ozone group than those in the control group at all post-operative follow-up time-points $(\mathrm{P}<0.05$; Table II; Figs. 2-4). The lower pain observed in the ozone group in the short term may be due to injection of Lidocaine into the joint during ozone administration. Of note, those differences were maintained even at 1 year post surgery. With regard to pain intensity, it was observed that the VAS scores significantly decreased from 7.52 prior to treatment to 5.17 at the 12-month follow-up in the ozone group, whereas in the control group, the VAS scores decreased from 7.66 to 6.56 (Table II; Fig. 2). The Lequesne Index improved from 15.07 to 8.74 in the ozone group and from 14.26 to 10.08 in the control group at the 12-month follow-up (Table II; Fig. 3). Furthermore, the WOMAC-pain, WOMAC-stiffness and WOMAC-function scores for the ozone group were significantly decreased from
12.36 to $6.71,2.95$ to 2.14 and 55.12 to 32.76 , respectively. In the control group, the same scores decreased from 15.9 to $4.4,3.32$ to 2.68 and 57.45 to 37.87 , respectively. The total WOMAC score decreased from 70.43 to 41.19 in the ozone group, and from 73.68 to 58.84 in the control group at the 12-month follow-up (Table II; Fig. 4). No significant difference in the WOMAC-stiffness score was observed after surgery at the 6 and 12-month follow-up assessments compared with pretreatment in the control group (Table II).

CGI scores. At the end of the follow-up of at least 12 months, based on the CGI, the treatment outcome was rated as excellent in $10(23.8 \%)$, good in $17(40.5 \%)$, satisfactory in $8(19.0 \%)$, unchanged in $4(9.5 \%)$ and worse in $3(7.1 \%)$ patients in the ozone group. In the control group, the treatment outcome was rated as excellent in $5(13.2 \%)$, good in $12(31.6 \%)$, satisfactory in $10(26.3 \%)$, unchanged in $7(18.4 \%)$ and worse in $4(10.5 \%)$ patients (Fig. 5). Analysis of the physician's CGI scores at the 12-month follow-up indicated that a significantly higher number of patients in the ozone group had been assigned better CGI grades (excellent and good) compared with that in the control group $(\mathrm{P}<0.05)$.

\section{Discussion}

Knee OA is a degenerative disease that leads to painful joints, articular stiffness and decreased function. At present, no cure is available for knee OA, and the major objectives of treatment are the relief of symptoms, including pain, stiffness and swelling, improvement of function and limitation of joint damage leading to the destruction of the joint. The pathophysiology of knee OA is complex, and is characterized by the wearing of the articular cartilage with subsequent damage to the bones and increased matrix degradation. Most orthopedic surgeons include arthroscopic surgery as a treatment modality for patients with mild or moderate knee OA. Arthroscopic surgery for knee OA 
Table I. Comparison of demographic data as well as VAS, Lequesne and WOMAC scores at baseline between the Ozone and Control groups.

\begin{tabular}{|c|c|c|c|}
\hline Parameter & Ozone group $(n=42)$ & Control group $(\mathrm{n}=38)$ & P-value \\
\hline $\operatorname{Age}^{\mathrm{a}}( \pm \mathrm{SD})$ & $68.83 \pm 5.56$ & $69.63 \pm 4.96$ & 0.502 \\
\hline Median (range) & $69(60-79)$ & $71(60-77)$ & \\
\hline $\operatorname{Sex}^{\mathrm{b}}$ & & & 0.408 \\
\hline Male & $17(40.5)$ & $12(31.6)$ & \\
\hline Female & $25(59.5)$ & $26(68.4)$ & \\
\hline Knee $^{\mathrm{b}}$ & & & 0.888 \\
\hline Right & $27(64.3)$ & $25(65.8)$ & \\
\hline Left & $15(35.7)$ & $13(34.2)$ & \\
\hline Knee OA grade ${ }^{\mathrm{b}}$ & & & 0.866 \\
\hline Grade II & $24(57.1)$ & $21(55.3)$ & \\
\hline Grade III & $18(42.9)$ & $17(44.7)$ & \\
\hline VAS (initial) ${ }^{\mathrm{a}}$ & $7.52 \pm 1.57$ & $7.66 \pm 1.32$ & 0.682 \\
\hline Lequesne (initial) ${ }^{\mathrm{a}}$ & $15.07 \pm 3.82$ & $14.26 \pm 2.64$ & 0.280 \\
\hline \multicolumn{4}{|l|}{ WOMAC (initial) ${ }^{\mathrm{a}}$} \\
\hline Pain & $12.36 \pm 2.79$ & $13.55 \pm 3.45$ & 0.091 \\
\hline Stiffness & $2.95 \pm 1.75$ & $3.32 \pm 2.04$ & 0.394 \\
\hline Physical function & $55.12 \pm 6.99$ & $57.45 \pm 6.16$ & 0.120 \\
\hline Total & $70.43 \pm 8.37$ & $73.68 \pm 10.07$ & 0.119 \\
\hline
\end{tabular}

${ }^{a}$ Independent-samples t-test; ${ }^{b}$ Pearson's Chi-square test. Values are expressed as n (\%) or the mean \pm standard deviation. WOMAC, Western Ontario and McMaster Universities OA Index; VAS, Visual Analogue Scale; OA, osteoarthritis.

may delay joint degeneration to maintain moderate activity and allow for future reconstruction, particularly for patients with mild to moderate OA $(21,22)$. However, the treatment cannot reverse or stop the pathological process of OA or prevent its continuous development. Therefore, its long-term effects are less than ideal. In the present study, it was also demonstrated that the efficacy of arthroscopic treatment for patients with knee OA of Kellgren-Lawrence Grade II or III had no obvious effect in joints stiffness after 6 months of follow-up in the control group. Treatment approaches combining arthroscopic surgery with viscosupplementation have been trialed to improve the outcomes of knee OA. For instance, treatment combining arthroscopic surgery and hyaluronic acid has been widely used. Hempfling (8) reported that the post-arthroscopic instillation of $0.5 \%$ sodium hyaluronate into the joint is a suitable method for achieving long-term stabilization of the treatment outcome. Mathies (7) performed a study examining the outcomes of the use of $0.5 \%$ sodium hyaluronate immediately after arthroscopic surgery. This study indicated that the use of $0.5 \%$ sodium hyaluronate may be of benefit in reducing post-operative pain and swelling. In addition, Huskin et al (23) reported that viscosupplementation with hylan G-F 20 had a favorable risk-benefit profile in patients with symptomatic knee OA after arthroscopic meniscectomy and/or debridement of the knee in a patient with chondral disease.

Although ozone has been widely used for the treatment of orthopedic diseases, at present there is no unanimous protocol for ozone treatment in knee OA $(12,14,24)$. It is a gas that is highly soluble in biological fluids, where atomic oxygen acts as a highly reactive species. After injection into a joint capsule, ozone is able to reduce inflammation, improve function and benefit the trophism, vascularization and repair of articular and subchondral bone. The biological mechanism of action of ozone therapy for knee OA is thought to be based on the biological effects elicited by ozone, including reactive oxygen species (ROS) and lipid oxidation products (LOPs) (12-14). It is considered that ROS and LOPs may inhibit the release of proteolytic enzymes or proinflammatory cytokines and stimulate the proliferation of fibroblasts and chondrocytes with increased synthesis of the matrix and articular cartilage (25). A randomized controlled trial comparing intra-articular injection of platelet-rich plasma, hyaluronic acid and ozone into the knee of OA patients indicated comparable effects on pain reduction among the three approaches after 1 month of treatment (26). Hashemi et al (18) reported that there was no significant difference in VAS and WOMAC scores between intra-articular dextrose and ozone treatment in patients with mild to moderate knee OA in the short term. Recently, Chansoria et al (27) indicated that in a randomized controlled trial, intra-articular injection of a combination of ozone and steroids in patients with primary knee OA resulted in a better outcome in terms of pain relief and functional improvements than that achieved with ozone alone. Several studies have indicated that intra-articular ozone injection for knee OA is a safe and effective treatment with a low risk of complications. In a single-blinded, controlled study comprising 84 patients, Invernizzi et al (28) demonstrated that in terms of safety, intra-articular ozone 
Table II. VAS, Lequesne and WOMAC scores in the Ozone and Control groups at Baseline, at 6 weeks and at 3,6 and 12 months after treatment.

\begin{tabular}{|c|c|c|c|c|c|c|}
\hline \multirow[b]{2}{*}{ Time-point/group } & \multirow[b]{2}{*}{ VAS } & \multirow[b]{2}{*}{ Lequesne } & \multicolumn{4}{|c|}{ WOMAC } \\
\hline & & & Pain & Stiffness & Physical function & Total \\
\hline \multicolumn{7}{|l|}{ Baseline } \\
\hline Ozone group & $7.52 \pm 1.57$ & $15.07 \pm 3.82$ & $12.36 \pm 2.79$ & $2.95 \pm 1.75$ & $55.12 \pm 6.99$ & $70.43 \pm 8.37$ \\
\hline Control group & $7.66 \pm 1.32$ & $14.26 \pm 2.64$ & $13.55 \pm 3.45$ & $3.32 \pm 2.04$ & $57.45 \pm 6.16$ & $73.68 \pm 10.07$ \\
\hline P-value ${ }^{\mathrm{a}}$ & 0.682 & 0.280 & 0.091 & 0.394 & 0.120 & 0.119 \\
\hline \multicolumn{7}{|l|}{6 weeks } \\
\hline Ozone group & $3.69 \pm 1.14$ & $5.86 \pm 2.40$ & $4.90 \pm 2.25$ & $1.67 \pm 1.12$ & $28.55 \pm 5.00$ & $35.12 \pm 6.23$ \\
\hline Control group & $4.53 \pm 1.67$ & $7.89 \pm 2.71$ & $6.97 \pm 2.45$ & $2.42 \pm 1.31$ & $33.84 \pm 6.26$ & $43.24 \pm 7.08$ \\
\hline P-value & 0.010 & 0.001 & $<0.001$ & 0.008 & $<0.001$ & $<0.001$ \\
\hline P-value ${ }^{b}$ & $<0.001$ & $<0.001$ & $<0.001$ & $<0.001$ & $<0.001$ & $<0.001$ \\
\hline P-value & $<0.001$ & $<0.001$ & $<0.001$ & 0.014 & $<0.001$ & $<0.001$ \\
\hline \multicolumn{7}{|l|}{3 months } \\
\hline Ozone group & $3.81 \pm 1.57$ & $6.02 \pm 2.05$ & $5.69 \pm 2.32$ & $2.00 \pm 1.12$ & $28.98 \pm 7.35$ & $36.67 \pm 8.11$ \\
\hline Control group & $4.74 \pm 1.54$ & $8.97 \pm 2.14$ & $7.71 \pm 2.04$ & $2.61 \pm 1.33$ & $34.82 \pm 7.61$ & $45.29 \pm 8.51$ \\
\hline P-value ${ }^{a}$ & 0.009 & 0.046 & $<0.001$ & 0.034 & $<0.001$ & $<0.001$ \\
\hline P-value ${ }^{b}$ & $<0.001$ & $<0.001$ & $<0.001$ & 0.002 & $<0.001$ & $<0.001$ \\
\hline P-value ${ }^{c}$ & $<0.001$ & $<0.001$ & $<0.001$ & 0.049 & $<0.001$ & $<0.001$ \\
\hline \multicolumn{7}{|l|}{6 months } \\
\hline Ozone group & $4.71 \pm 1.42$ & $7.19 \pm 2.24$ & $5.83 \pm 1.81$ & $2.25 \pm 1.47$ & $29.95 \pm 6.67$ & $38.02 \pm 7.66$ \\
\hline Control group & $5.97 \pm 1.23$ & $9.21 \pm 2.04$ & $7.95 \pm 1.90$ & $2.76 \pm 1.56$ & $36.50 \pm 8.77$ & $47.00 \pm 8.70$ \\
\hline P-value ${ }^{a}$ & $<0.001$ & 0.037 & $<0.001$ & 0.042 & $<0.001$ & $<0.001$ \\
\hline$P$-value ${ }^{b}$ & $<0.001$ & $<0.001$ & $<0.001$ & 0.021 & $<0.001$ & $<0.001$ \\
\hline P-value & $<0.001$ & $<0.001$ & $<0.001$ & 0.126 & $<0.001$ & $<0.001$ \\
\hline \multicolumn{7}{|l|}{12 months } \\
\hline Ozone group & $5.17 \pm 1.43$ & $8.74 \pm 2.89$ & $6.71 \pm 2.38$ & $2.33 \pm 1.37$ & $32.76 \pm 6.64$ & $41.19 \pm 7.64$ \\
\hline Control group & $6.76 \pm 1.45$ & $10.08 \pm 3.03$ & $8.55 \pm 2.44$ & $2.68 \pm 1.47$ & $37.87 \pm 6.96$ & $58.84 \pm 7.48$ \\
\hline P-value & $<0.001$ & 0.046 & $<0.001$ & 0.047 & 0.001 & $<0.001$ \\
\hline $\mathrm{P}$-value ${ }^{\mathrm{b}}$ & $<0.001$ & $<0.001$ & $<0.001$ & 0.032 & $<0.001$ & $<0.001$ \\
\hline P-value & 0.008 & $<0.001$ & $<0.001$ & 0.080 & $<0.001$ & $<0.001$ \\
\hline
\end{tabular}

${ }^{\mathrm{a}}$ Ozone vs. control groups at the same time points. ${ }^{\mathrm{b} O z o n e}$ vs. baseline ozone group. ${ }^{\mathrm{c}}$ Ozone vs. the baseline control group. Values are expressed as the mean \pm standard deviation. WOMAC, Western Ontario and McMaster Universities Osteoarthritis Index; VAS, Visual Analogue Scale.

treatment is comparable to intra-articular sodium hyaluronate in chronic knee OA. In the present study, no serious complications occurred in the ozone treatment group. Only 5 patients felt a mild pain after the injection, which disappeared within a few minutes. This side effect was also reported by Lopes de Jesus et al (29).

The present study revealed that ozone treatment reduced pain, as measured by the VAS. It also improved the quality of life measured by the Lequesne Index score and total WOMAC score in the ozone group. These significant changes demonstrate the effectiveness of ozone therapy in reducing pain, and improving function and quality of life of these patients post-surgery. The positive results of intra-articular ozone injection for knee OA are further supported the results of Lopes de Jesus et al (29). Another recent study by Fernández-Cuadros et al (30) indicated that ozone treatment decreases the VAS, as well as the WOMAC-pain,
WOMAC-stiffness and WOMAC-function scores in patients with knee OA. The present study also indicated that the ozone group had a significantly greater improvement in pain and function than the control group, according to the VAS, Lequesne index and WOMAC function scores. Based on the CGI score at the 12-month follow-up, $64.3 \%$ of the patients obtained excellent or good results in the ozone group. By contrast, only $42.1 \%$ of the patients obtained excellent or good results in the control group. The reason for the favorable long-term effects in the ozone group may be that ozone promotes better vascularization in bones and cartilage, and accelerating anabolism and recovery in osteoarticular diseases (12). However, the exact mechanism of action of ozone therapy in knee OA has remained to be fully elucidated, and further studies are required to confirm the results and the long-term outcome.

The present study had several limitations. First, it was limited by the small sample size, and lidocaine was not 


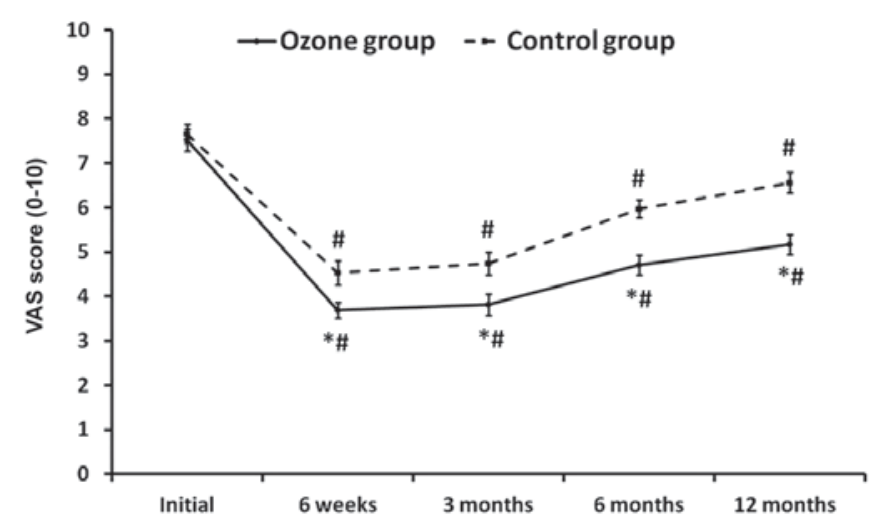

Figure 2. VAS scores in the groups pre- and post-treatment. "P<0.05 vs. control group; ${ }^{*} \mathrm{P}<0.05$ vs. baseline. VAS, Visual Analogue Scale.

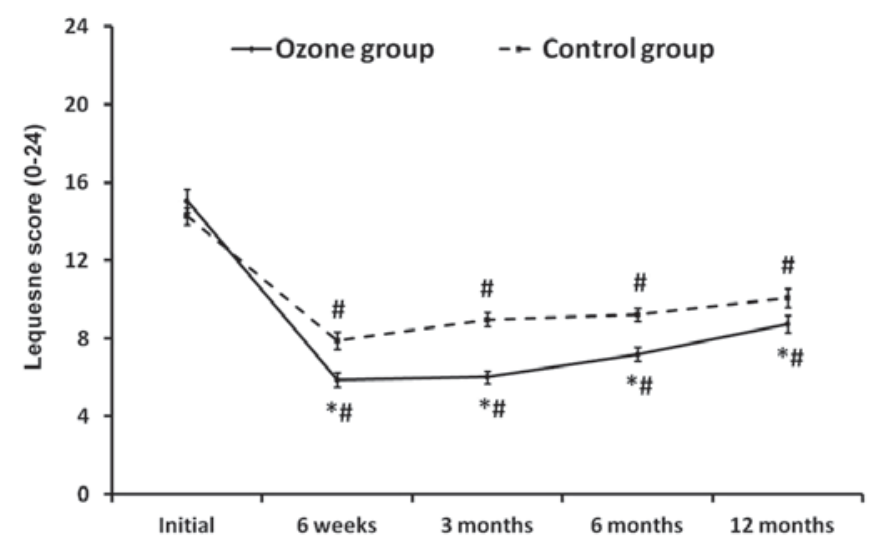

Figure 3. Lequesne scores in the groups pre- and post-treatment. " $\mathrm{P}<0.05$ vs. control group; ${ }^{\sharp \mathrm{P}}<0.05$ vs. baseline.

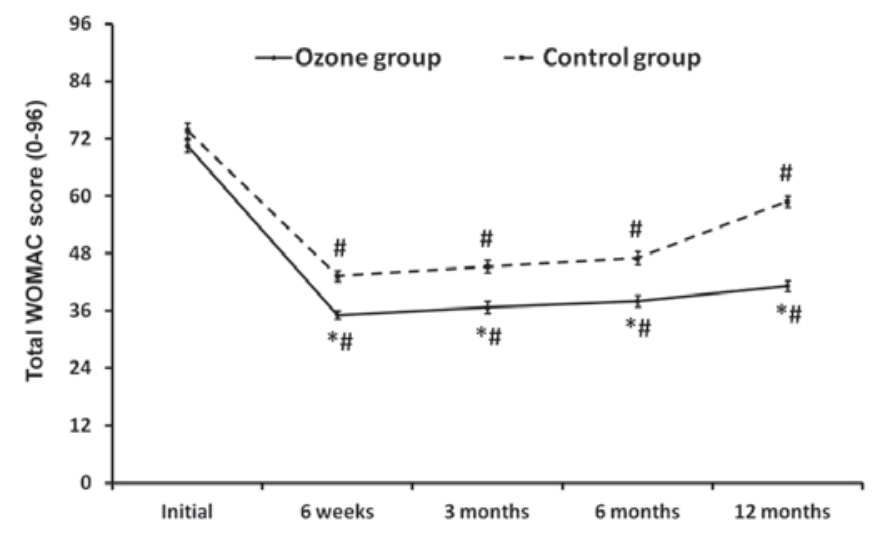

Figure 4. Total WOMAC scores in the groups pre- and post-treatment. ${ }^{*} \mathrm{P}<0.05$ vs. control group; ${ }^{\#} \mathrm{P}<0.05$ vs. baseline. WOMAC, Western Ontario and McMaster Universities Osteoarthritis Index.

injected into the knee in the control group, which may have affected the results. It will be necessary to expand the sample size and perform rigorous prospective studies in the future. Furthermore, the present study only recruited patients with Kellgren-Lawrence grade II or III OA, so the results are difficult to generalize to all OA populations that have different degrees of radiographically evident severity. In addition, the present study only investigated the clinical

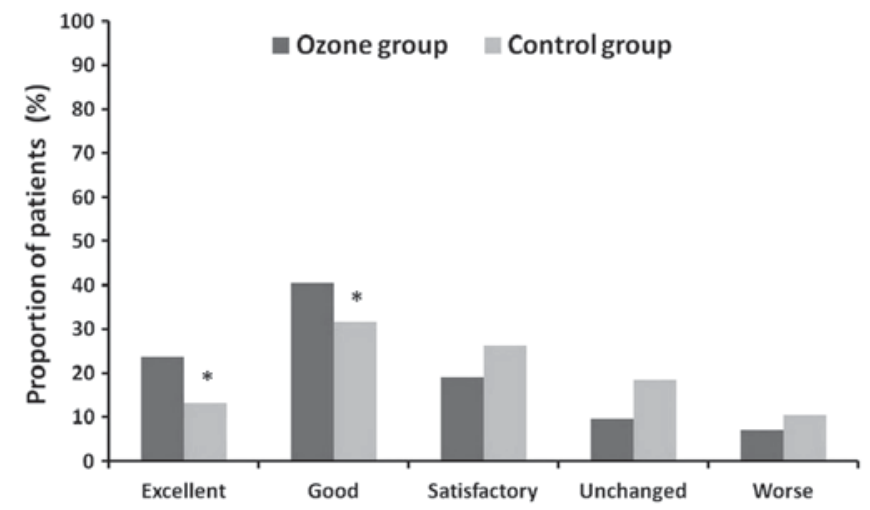

Figure 5. Clinical Global Impression by patients in the two groups at the 12-month follow-up. ${ }^{*} \mathrm{P}<0.05$ vs. control group.

symptomatic changes, and no imaging features of knee OA were studied.

In conclusion, the results of the present study demonstrated the efficacy of intra-articular ozone injections after arthroscopic surgery in relieving pain and improving physical function in knee OA of Kellgren-Lawrence grade II or III. However, the present results should be looked at with caution and require further validation in larger cohorts.

\section{Acknowledgements}

The authors wish to thank Yingchao Han from the Department of Orthopedics (Renji Hospital, Shanghai Jiao Tong University School of Medicine, China) for performing the statistical analysis.

\section{Funding}

This study was funded by the Sixth Affiliated Hospital of Guangzhou Medical University (Guangzhou, China; grant no. A2016222).

\section{Availability of data and materials}

The analyzed data sets generated during the study are available from the corresponding author on reasonable request.

\section{Authors' contributions}

DC and XW conceived and designed the study. XW, GW and CL performed the experiments. XW wrote the paper. DC, XW, GW and CL reviewed and edited the manuscript. XW and CL analysed the data. All authors read and approved the manuscript and agree to be accountable for all aspects of the research in ensuring that the accuracy or integrity of any part of the work are appropriately investigated and resolved.

\section{Ethical approval and consent to participate}

This study was approved by the Ethics Committee of the Sixth Affiliated Hospital of Guangzhou Medical University (Guangzhou, China) and performed in accordance with the provisions of the World Medical Association Declaration 
of Helsinki 1995 (revised in Tokyo, 2004). Due to the retrospective nature of the study, the Ethics Committee of the Sixth Affiliated Hospital of Guangzhou Medical University (Guangzhou, China) waived the requirement for study participants to provide informed consent.

\section{Consent for publication}

Not applicable.

\section{Competing interests}

The authors declare that they have no competing interests.

\section{References}

1. M Hawamdeh Z and Al-Ajlouni JM: The clinical pattern of knee osteoarthritis in jordan: A hospital based study. Int J Med Sci 10: 790-795, 2013

2. Pearse EO and Craig DM: Partial meniscectomy in the presence of severe osteoarthritis does not hasten the symptomatic progression of osteoarthritis. Arthroscopy 19: 963-968, 2003.

3. Hunt SA, Jazrawi LM and Sherman OH: Arthroscopic management of osteoarthritis of the knee. J Am Acad Orthop Surg 10 356-363, 2002

4. Jackson RW and Dieterichs C: The results of arthroscopic lavage and debridement of osteoarthritic knees based on the severity of degeneration: A 4- to 6-year symptomatic follow-up. Arthroscopy 19: 13-20, 2003.

5. Figueroa D, Calvo R, Villalón IE, Meleán P, Novoa F and Vaisman A: Clinical outcomes after arthroscopic treatment of knee osteoarthritis. Knee 20: 591-594, 2013.

6. Forster MC and Straw R: A prospective randomized trial comparing intra-articular hyalgan injection and arthroscopic washout for knee osteoarthritis. Knee 10: 291-293, 2003.

7. Mathies B: Effects of viscoseal, a synovial fluid substitute, on recovery after arthroscopic partial meniscectomy and joint lavage. Knee Surg Sports Traumatol Arthrosc 14: 32-39, 2006.

8. Hempfling $\mathrm{H}$ : Intra-articular hyaluronic acid after knee arthroscopy: A two-year study. Knee Surg Sports Traumatol Arthrosc 15: 537-546, 2007.

9. Ulucay C, Altintas F, Ugutmen E and Beksaç B: The use of arthroscopic debridement and viscosupplementation in knee osteoarthritis. Acta Orthop Traumatol Turc 41: 337-342, 2007 (In Turkish).

10. Zietz PM and Selesnick H: The use of hylan G-F 20 after knee arthroscopy in an active patient population with knee osteoarthritis. Arthroscopy 24: 416-422, 2008

11. Bocci V: Ozone as Janus: This controversial gas can be either toxic or medically useful. Mediators Inflamm 13: 3-11, 2004

12. Fernandez-Cuadros ME, Perez-Moro OS and Mirón-Canelo JA: Could ozone be used as a feasible future treatment in osteoarthritis of the knee? Diversity Equal Health Care 13: 232-239, 2016.

13. Swapan KM, Rajesh P, Pallab D, Partha PD, Asim KP, Jayanta R, Halder $\mathrm{N}$ and Rathindra NH: Role of intra-articular ozone in osteo-arthritis of knee for functional and symptomatic improvement. Ind J Phys Med Rehabil 22: 65-69, 2011.

14. Borrelli E, Alexandre A, Iliakis E, Alexandre A and Bocci V: Disk herniation and knee arthritis as chronic oxidative stress disieases: The therapeutic role of oxygen ozone therapy. J Arthritis 4: 161, 2015.
15. Zhang JF, Zhan XQ, Hu HW, Yang Q and Wang XH: Clinical results of medical ozone combined with sodium hyaluronate for knee osteoarthritis. Orthop J China 5: 362-365, 2010 (In Chinese)

16. Kellgren JH and Lawrence JS: Radiological assessment of osteo arthrosis. Ann Rheum Dis 16: 494-502, 1957.

17. Price DD, McGrath PA, Rafii A and Buckingham B: The validation of visual analogue scales as ratio scale measures for chronic and experimental pain. Pain 17: 45-56, 1983.

18. Hashemi M, Jalili P, Mennati S, Koosha A, Rohanifar R, Madadi F, Razavi SS and Taheri F: The effects of prolotherapy with hypertonic dextrose versus prolozone (intraarticular ozone) in patients with knee osteoarthritis. Anesth Pain Med 5: e27585, 2015.

19. Lequesne MG: The algofunctional indices for hip and knee osteoarthritis. J Rheumatol 24: 779-781, 1997.

20. Bellamy N, Buchanan WW, Goldsmith $\mathrm{CH}$, Campbell J and Stitt LW: Validation study of WOMAC: a health status instrument for measuring clinically important patient relevant outcomes to antirheumatic drug therapy in patients with osteoarthritis of the hip or knee. J Rheumatol 15: 1833-1840, 1988.

21. Aaron RK, Skolnick AH, Reinert SE and Ciombor DM: Arthroscopic debridement for osteoarthritis of the knee. J Bone Joint Surg Am 88: 936-943, 2006.

22. Shin CS and Lee JH: Arthroscopic treatment for osteoarthritic knee. Knee Surg Relat Res 24: 187-192, 2012.

23. Huskin JP, Vandekerckhove B, Delincé P, Verdonk R, Dubuc JE, Willems S, Hardy P, Blanco FJ, Charrois $\mathrm{O}$ and Handelberg F: Multicentre, prospective, open study to evaluate the safety and efficacy of hylan G-F 20 in knee osteoarthritis subjects presenting with pain following arthroscopic meniscectomy. Knee Surg Sports Traumatol Arthrosc 16: 747-752, 2008.

24. Calunga J L, Menéndez S, León R, Chang S, Guanche D, Balbín A, Zayas J and García P: Application of ozone therapy in patients withknee osteoarthritis. Ozone: Science \& Engineering 34: 469-475, 2012

25. Bocci VA: Scientific and medical aspects of ozone therapy. State of the art. Arch Med Res 37: 425-435, 2006.

26. Duymus TM, Mutlu S, Dernek B, Komur B, Aydogmus S and Kesiktas FN: Choice of intra-articular injection in treatment of knee osteoarthritis: Platelet-rich plasma, hyaluronic acid or ozone options. Knee Surg Sports Traumatol Arthrosc 25: 485-492, 2017.

27. Chansoria M, Upadhyay S, Panwar S, Shivhare P and Vyas N: Comparative efficacy of intraarticular injection of combination of ozone and steroid and ozone alone in patients with primary knee osteoarthritis: A prospective, randomized clinical analysis. J Recent Adv Pain 2: 11-14, 2016.

28. Invernizzi M, Stagno D, Carda S, Grana E, Picelli A, Smania N, Cisari $\mathrm{C}$ and Baricich A: Safety of intra-articular oxygen-ozone therapy compared to intra-articular sodium hyaluronate in knee osteoarthritis: A randomized single blind pilot study. Int J Phys Med Rehabil 5: 385, 2017.

29. Lopes de Jesus CC, Dos Santos FC, de Jesus LMOB, Monteiro I, Sant'Ana MSSC and Trevisani VFM: Comparison between intra-articular ozone and placebo in the treatment of knee osteoarthritis: A randomized, double-blinded,placebo-controlled study. PLoS One 12: e0179185, 2017.

30. Fernández-Cuadros ME, Pérez-Moro OS, Albaladejo-Florin MJ, et al: Ozone improves pain, function and quality of life in patients with knee osteoarthritis: A prospective quasi-experimental before-after study. Middle East J Rehabil Health 4: e41821, 2017.

cc) (i) $\ominus$ This work is licensed under a Creative Commons Attribution-NonCommercial-NoDerivatives 4.0 International (CC BY-NC-ND 4.0) License. 